\title{
DOCUMENTATION OF TOXICITY TESTING RESULTS ON INCREASED SUPERNATE TREATMENT RATE OF 2700 GALLONS/BATCH(U)
}

by

\author{
J. B. Pickett
}

Westinghouse Savannah River Company

Savannah River Site

Aiken, South Carolina 29808

\section{DISCLAIMER}

\begin{abstract}
This report was prepared as an account of work sponsored by an agency of the United States This report was prepared as an account of work spont nor any agency thereof, nor any of their employees, makes any warranty, express or implied, or assumes any legal liability or responsibility for the accuracy, completeness, or usefulness of any information, apparatus, product, or process disclosed, or represents that its use would not infringe privately owned rights. Reference herein to any specific commercial product, process, or service by trade name, trademark, manufacturer, or otherwise does not necessarily constitute or imply its endorsement, recommendation, or favoring by the United States Government or any agency thereof. The views and opinions of authors expressed herein do not necessarily state or reflect those of the United States Government or any agency thereof.
\end{abstract}

This report was prepared in connection with work done under Contract No. DE-AC09-89SR18035 with the U.S. Department of Energy. By acceptance of this report, the publisher and/or recipient acknowledges the U.S. Government's right to retain a nonexclusive, royalty-free license in and to any copyright covering this report, along with the right to reproduce and to authorize others to reproduce all or part of the copyrighted report. 


\section{WESTINGHOUSE SAVANNAH RIVER COMPANY INTER-OFFICE MEMORANDUM

September 16, 1991

Revised July 6, 1992

TO. C. P. Thompson, 730-M

Reactor Materials Engineering and Technology

FROM: J. B. Pickett. 730-M (5-3838)

Reactor Materials Engineering and Technology

\section{Documentation of Toxicity Testing Results on \\ Increased Supernate Treatment Rate of 2700 Gallons/Batch (U)}

Summary

In February 1991, Reactor Materials increased the rate of supernate treatment in the M-Area Dilute Effluent Treatment Facility (DETF) from 1800 gallons to 22700 gallons of supemate per 36,000 gallon dilute wastewater batch. The first release of the treated effuent began March 3, 1991. A series of whole effluent toxicity tests was conducted on the DETF effluent to determine if the increased supernate concentration would" result in any chronic toxicity affects in the receiving stream (Tims Branch). The' toxicity tests were conducted at insiream concentrations equivalent to DETF release rates of $5,10,15,20$, and 25 gallons/min.

The test results, based on 7-day Ceriodaphniadubia chronic toxicity, indicated no toxicity effects at any concentration tested. Supernate treatment in the DETF continued at the higher concentration. The toxicity results are summarized below:

Toxicity Results: Average No. Young

$\begin{array}{llllll}\text { Control ( } i \text { of Young) } & 16.9 & 19.4 & 21.8 & 20.0 & 21.0 \\ \text { Test (\# of Young) } & 18.6 & 20.0 & 20.2 & 15.0 & 22.2 \\ \text { Test Different From Control } & \text { No } & \text { No } & \text { No } & \text { No } & \text { No } \\ \begin{array}{l}\text { Instream Concentration, \% } \\ \begin{array}{l}\text { Equivalent DETF } \\ \text { Release Rate, gpm }\end{array}\end{array} & 0.44 & 0.89 & 1.33 & 1.78 & 2.22 \\ \end{array}$




$\begin{array}{lccc}\text { Date } & 3 / 4 / 91 & 3 / 6 / 91 & 3 / 8 / 91 \\ \text { Time Sampled } & 0945 & 0900 & 0900 \\ \text { Flow rate, gpm } & 5 & 5 & 5 \\ \text { Collecilon location } & \text { M-004 } & \text { M-004 } & \text { M-004 } \\ \text { Nomandeau Log.No. } & \text { Ho0014 } & \text { NA } & \text { H00028 }\end{array}$

- Sample collection dates for Chronic Toxicity Test Renewals

(These samples were diluted by the testing laboratory to the concentrations equivalent to various DETF release rates)

7 Dey Chronle Toxicity Test Results .-

$20 \%$ Perrier/Milli Q water

Ave. No. Repro.

-Instream" concentration $\%$

Equivalent DETF release rate $\mathrm{gpm}$

Ave. No. Repro

$\begin{array}{ccccc}\text { Control } & \text { Control } & \text { Control } & \text { Control } & \text { Control } \\ 16.9 & 19.35 & 21.8 & 20 & 21 \\ 0.44 & 0.89 & 1.33 & 1.78 & 2.22 \\ 5 & 10 & 15 & 20 & 25 \\ 18.55 & 19.95 & 20.18 & 14.95 & 22.2 \\ \text { Pass } & \text { Pass } & \text { Pass } & \text { Pass } & \text { Pass }\end{array}$

Pass/Fail

Pass

Pass

Pass

Pass

Pass

\#Assuming $1125 \mathrm{gpm}(1.6 \mathrm{MGO})$ at A-014

-Toxicity testing by Normandeau Associates, New Ellenton, SC.

\begin{tabular}{|c|c|c|c|c|c|c|c|c|}
\hline & & Normandeau & Chomical & Analyses & & & & \\
\hline Samplo Id & $\overline{\%}$ & Control & $0.44^{\circ}$ & $0.89^{\circ}$ & $1.33^{\circ}$ & $1.78^{\circ}$ & $2.22^{\circ}$ & $100^{\circ}$ \\
\hline pH & & 7.70 & 7.61 & 7.78 & 7.79 & 7.83 & 7.84 & 7.27 \\
\hline Temp. & ${ }^{\circ} \mathrm{C}$ & 24.9 & 24.5 & 24.4 & 24.5 & 24.6 & 24.7 & $\cdot$ \\
\hline Alkalinity & (ppm Caco3) & 61.1 & . & - & - & - & - & 46.7 \\
\hline Hardness & (ppm CaCO3) & 78.9 & & - & . & - & - & 4.33 \\
\hline Conductivity & $(\mathrm{mS} / \mathrm{cm})$ & 0.167 & 0.221 & 0.273 & 0.323 & 0.372 & 0.419 & 9.83 \\
\hline Dissolved Oxygen & $\mathrm{mg} / \mathrm{L}$ & 7.70 & 7.65 & 7.68 & 7.58 & 7.6 & 7.54 & - \\
\hline Total Residual $\mathrm{Cl}$ & mgil & . & . & - & . & . & - & $<0.02$ \\
\hline
\end{tabular}

The chemical lab. results are the average of $7\left(^{\circ}\right)$ analyses and $3\left({ }^{\circ}\right)$ analyses, respctively on each of the "instream" concentration samples.

320-M Lab. Analyzes on Equalization Tank and Effluent Samples:

\begin{tabular}{|c|c|c|c|}
\hline & & $\begin{array}{l}\text { Equalization } \\
\text { Tank } \\
\end{array}$ & Ellluent: \\
\hline Date sampled & & $2 / 25 / 91$ & $3 / 1 / 91$ \\
\hline Time sampled & & 0255 & 0700 \\
\hline 320 Lab. Id. No. & & 351 & 398 \\
\hline pH & & 8.3 & 7.58 \\
\hline Tot. Suspended solids & $\mathrm{mg} / \mathrm{L}$ & 1335 & 5.0 \\
\hline Al & $\mathrm{mg} / \mathrm{L}$ & 52.1 & 0.047 \\
\hline$B$ & $\mathrm{mg} / \mathrm{L}$ & 0.29 & 2.01 \\
\hline $\operatorname{cd}$ & $\mathrm{mg} / \mathrm{L}$ & $<0.002$ & $<0.002$ \\
\hline $\mathrm{Cr}$ & $\mathrm{mg} / \mathrm{L}$ & 0.04 & $<0.002$ \\
\hline Cu & $\mathrm{mg} / \mathrm{L}$ & 0.004 & $<0.002$ \\
\hline$F_{\boldsymbol{\theta}}$ & $\mathrm{mg} / \mathrm{L}$ & 0.81 & 0.014 \\
\hline $\mathrm{Ni}$ & $\mathrm{mg} / \mathrm{L}$ & 0.07 & 0.002 \\
\hline Pb & $\mathrm{mg} / \mathrm{L}$ & 0.02 & $<0.013$ \\
\hline Sn & $\mathrm{mg} / \mathrm{L}$ & 0.03 & 0.009 \\
\hline $\mathrm{Zn}$ & $\mathrm{mg} / \mathrm{L}$ & 0.11 & 0.015 \\
\hline$u$ & $\mathrm{mg} / \mathrm{L}$ & $<0.01$ & 005 \\
\hline NITRATE (as N) & $\mathrm{mg} / \mathrm{L}$ & 1291.0 & 1002.0 \\
\hline PHOSPHATE (as P) & $\mathrm{mg} / \mathrm{L}$ & 21.0 & 12.8 \\
\hline CHLORIDE & $\mathrm{mg} / \mathrm{L}$ & 20.6 & \\
\hline SULFATE(as S) & $\mathrm{mg} / \mathrm{L}$ & 2859 & . \\
\hline
\end{tabular}

\#A grab sample trom the DETF storage tank. 
Normendewe Areacletes, Inc.

PO. BOX 1393

Aiken. SC 29802

(803) 652-2206

(8003)652-7428 (Fax)

6 April 1991

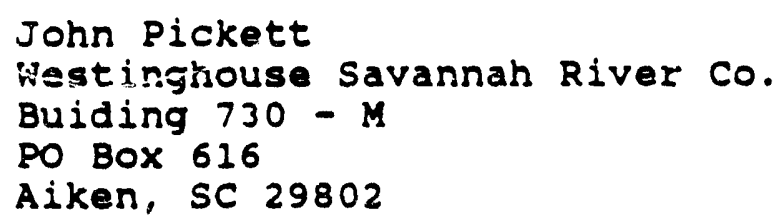

Dear John:

Enclosed please find copies of tables summarizing the results' be the 5 SC Pass/Fail chronic toxicity tests, a copy of a SC chronic toxicity test data form, and your chain-or-custody forms. This contract specifies that we perform only sc chronic toxicity tests and the price we quoted reflects the level of effort associated with performing and reporting these test results. The tables that I've submitted are adopted from those used by the state of South Carolina to summarize these tests. I've provided the chemistry data in more detail than would have appeared on the SC form; shemistry data is summarized in Tables 6 and 7 . As you can see, no chronic effect was indicated at any of the concentrations tested.

Please contact me if I can answer any questions you might have regarding these data.

Yours,

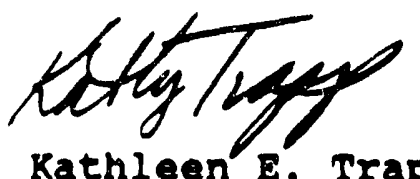

Kathleen E. Trapp, Ph.D.

General Manager

Normandeau Associates

Aiken, SC Greenville SC LeClarre. IA 
Table 1. Summary of observations made during a 7-day ceriodaphnia dubia chronic toxicity test performed on an effluent collected from the M004 outfall located on the Savannah River site. Laboratory water (20) Perrier/Milli-Q water) served as the control and diluent for this test. A 0.448 effluent solution was tested. 5 - 12 March 1991.

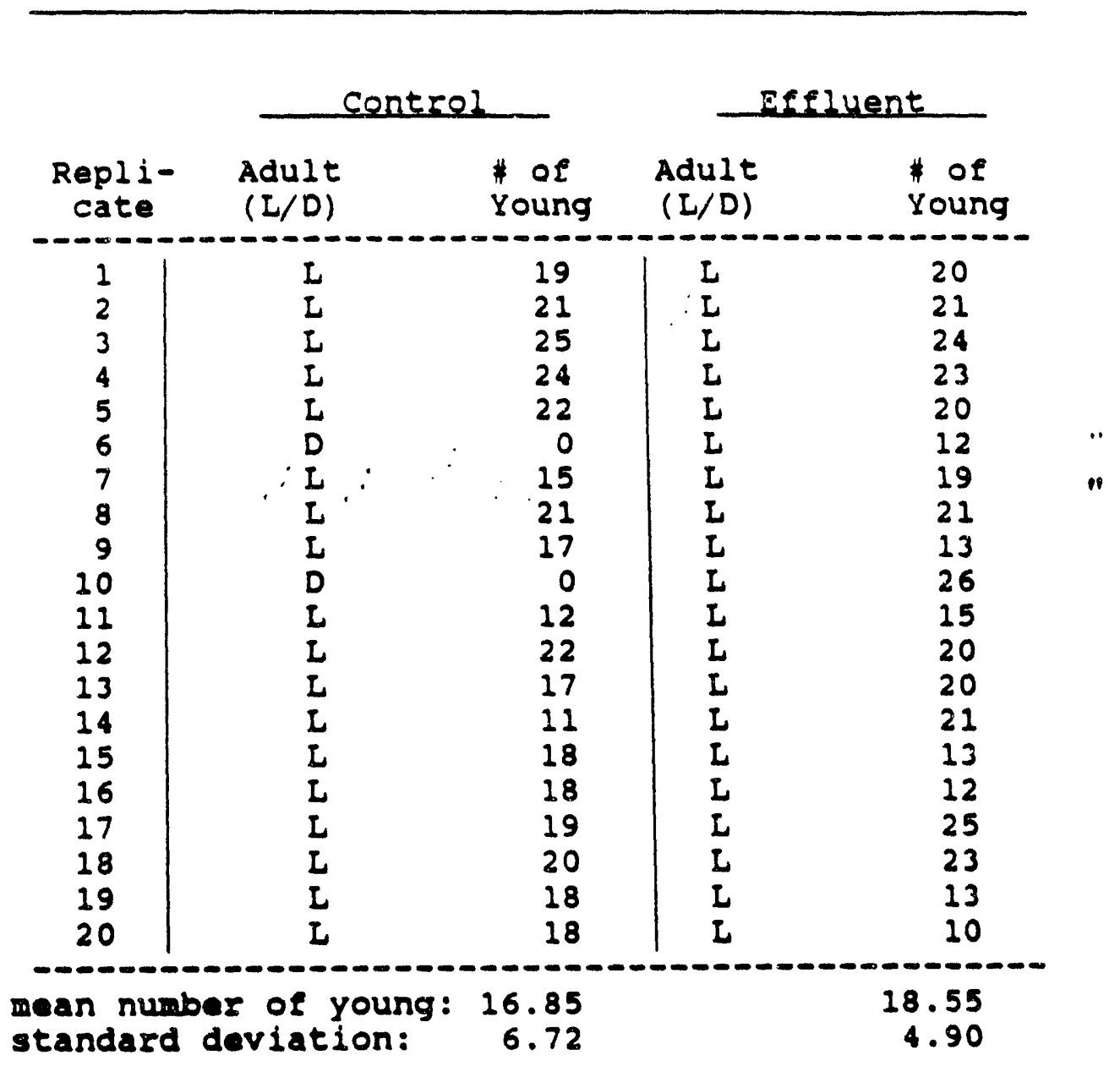

Pan LFaid Renulte Control:

0.447 Eefluent: Statistical Test: Pass/Fail:
SMortality 10 Fisher's Pass
Young Prod. 16.85 18.55

NA Pass 
Table 3. Summary of observations made during a 7-day Ceriodaphnia dubia chronic toxicity test performed on an effluent collected from the M004 outfall located on the Savannah River Site. Laboratory water (20t Perrier/Milli-Q water) served as the control and diluent for this test. A 1.33 effluent solution was tested. 5 - 12 March 1991.

\begin{tabular}{|c|c|c|c|c|}
\hline \multirow[b]{2}{*}{$\begin{array}{l}\text { Repli- } \\
\text { cate }\end{array}$} & \multicolumn{2}{|c|}{ centrel } & \multicolumn{2}{|c|}{ EEEluent } \\
\hline & $\begin{array}{l}\text { Adult } \\
(\mathrm{L} / \mathrm{D})\end{array}$ & $\begin{array}{l}\text { *of } \\
\text { young }\end{array}$ & $\begin{array}{l}\text { Adult } \\
(\mathrm{L} / \mathrm{D})\end{array}$ & $\begin{array}{l}\text { \# of } \\
\text { young }\end{array}$ \\
\hline $\begin{array}{r}1 \\
2 \\
3 \\
4 \\
5 \\
6 \\
7 \\
8 \\
9 \\
10 \\
11 \\
12 \\
13 \\
14 \\
15 \\
16 \\
17 \\
18 \\
19 \\
20\end{array}$ & 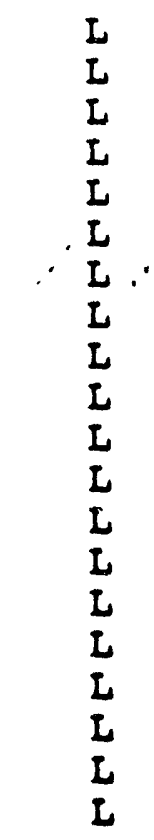 & $\begin{array}{l}18 \\
15 \\
23 \\
25 \\
25 \\
23 \\
26 \\
26 \\
20 \\
16 \\
24 \\
15 \\
23 \\
19 \\
26 \\
26 \\
25 \\
15 \\
22 \\
23\end{array}$ & $\begin{array}{l}L \\
L \\
L \\
L \\
L \\
L \\
L \\
L \\
L \\
L \\
L \\
L \\
L \\
I \\
L \\
L \\
L \\
L \\
L \\
L\end{array}$ & $\begin{array}{r}\text { MALE } \\
12 \\
13 \\
11 \\
\text { MAIE } \\
\text { MALE } \\
19 \\
26 \\
23 \\
23 \\
22 \\
18 \\
22 \\
23 \\
15 \\
22 \\
21 \\
24 \\
25 \\
24\end{array}$ \\
\hline $\begin{array}{l}\operatorname{can} n u \\
\text { tandar }\end{array}$ & or $y$ & $\begin{array}{r}21.75 \\
4.04\end{array}$ & & $\begin{array}{r}20.18 \\
4.72\end{array}$ \\
\hline
\end{tabular}

Pare/Padi Ronulte Control : 1.33 Efeluent: statistical Test: Pass/Fail:

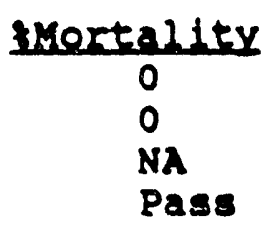

Young Prode

21.75

20.18

$t$-tent

pasa 
Table 5. Summary of observations made during a 7-day ceriodaphnia dubia chronic toxicity test performed on an effluent collected from the M004 outfall located on the Savannah River Site. Laboratory water (20t Perrier/Milli-Q water) served as the control and diluent for this test. A 2.22 effluent solution was tested. 5 - 12 March 1991.

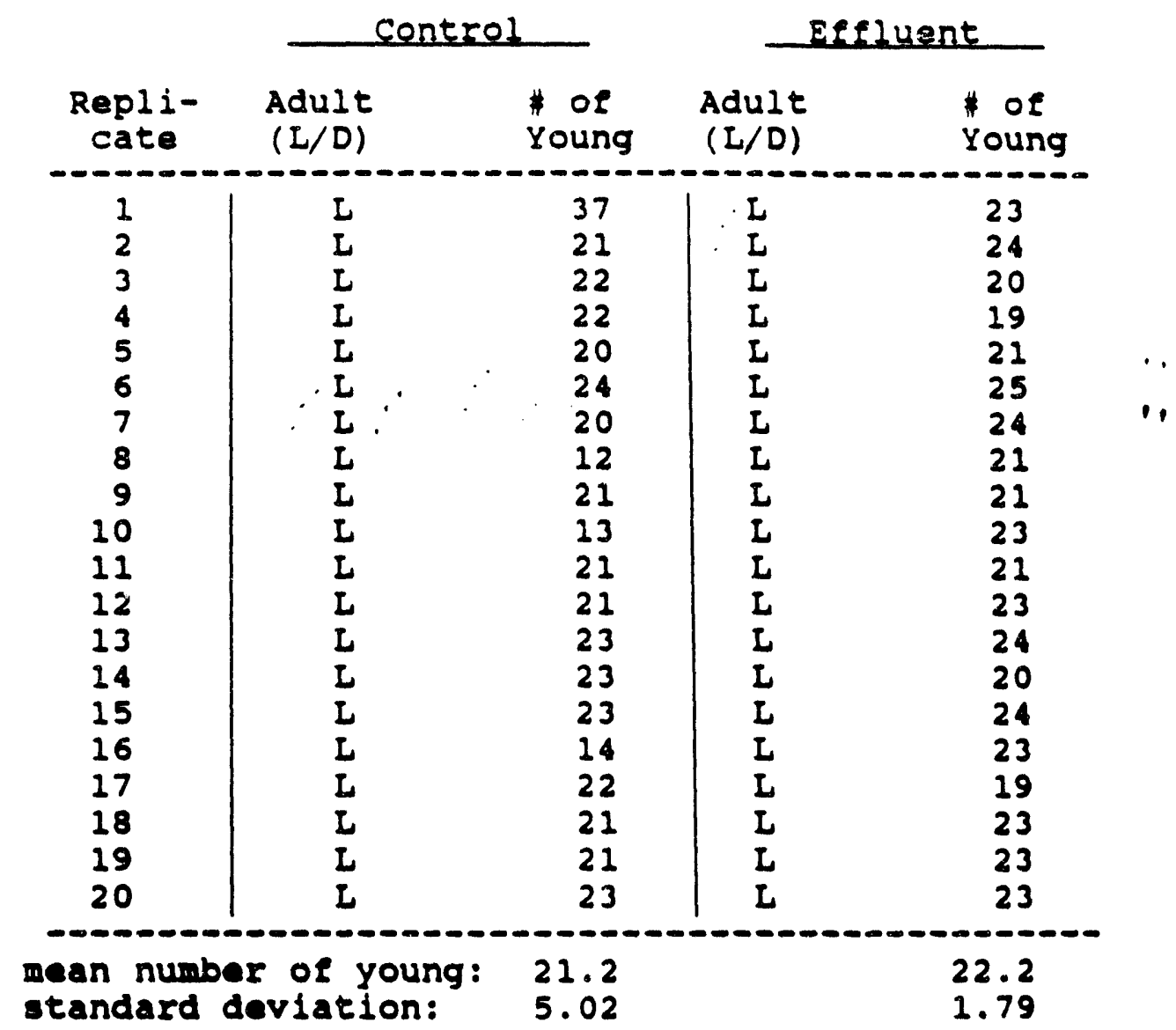

PandFall Renults

Control:

2.228 Effluent:

iMortality

0

statistical rest:

Fisher's

$\frac{\text { Young Prode }}{21.20}$

22.20

Pass/Fail:

Pass

NA

Pass 


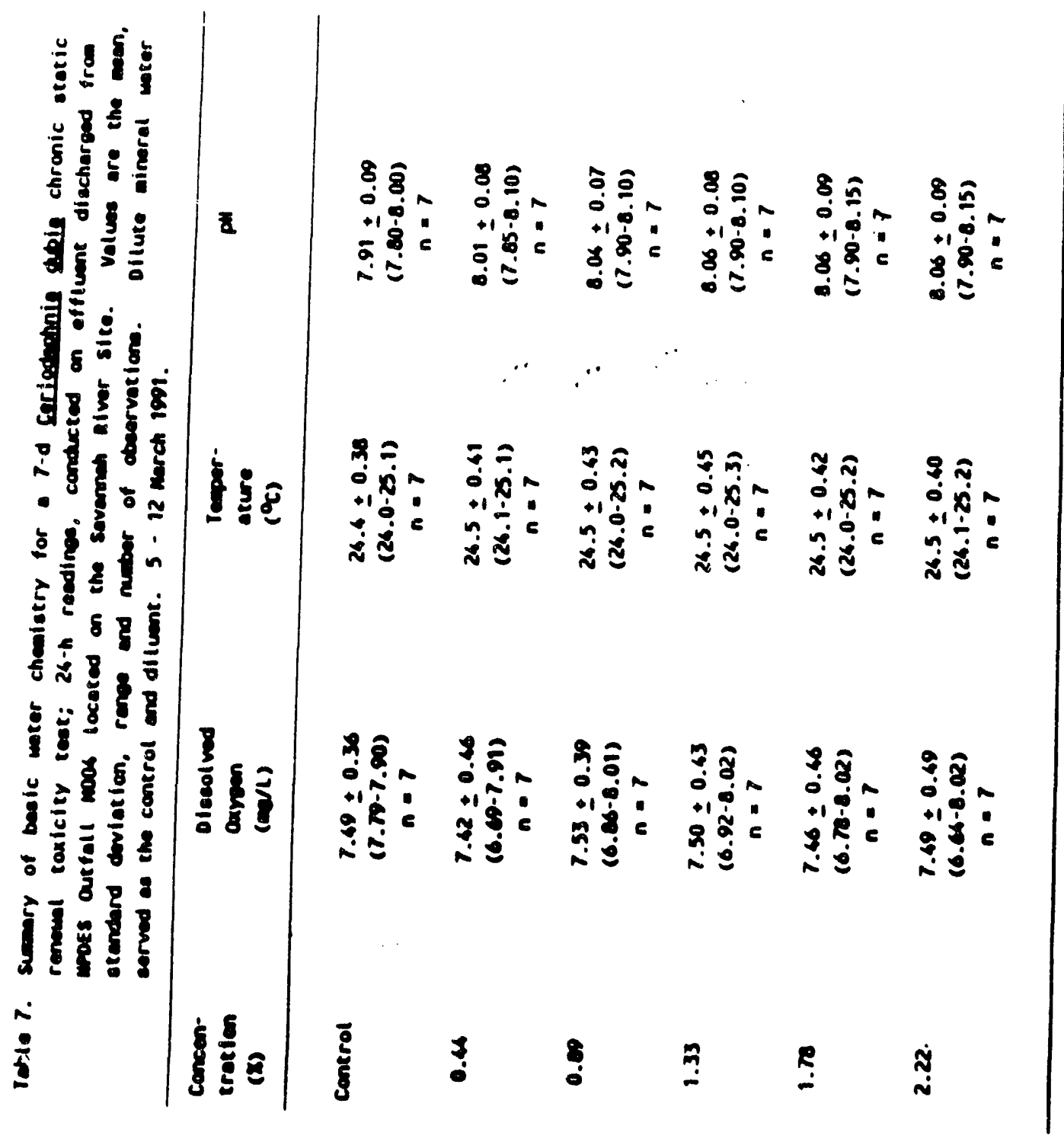




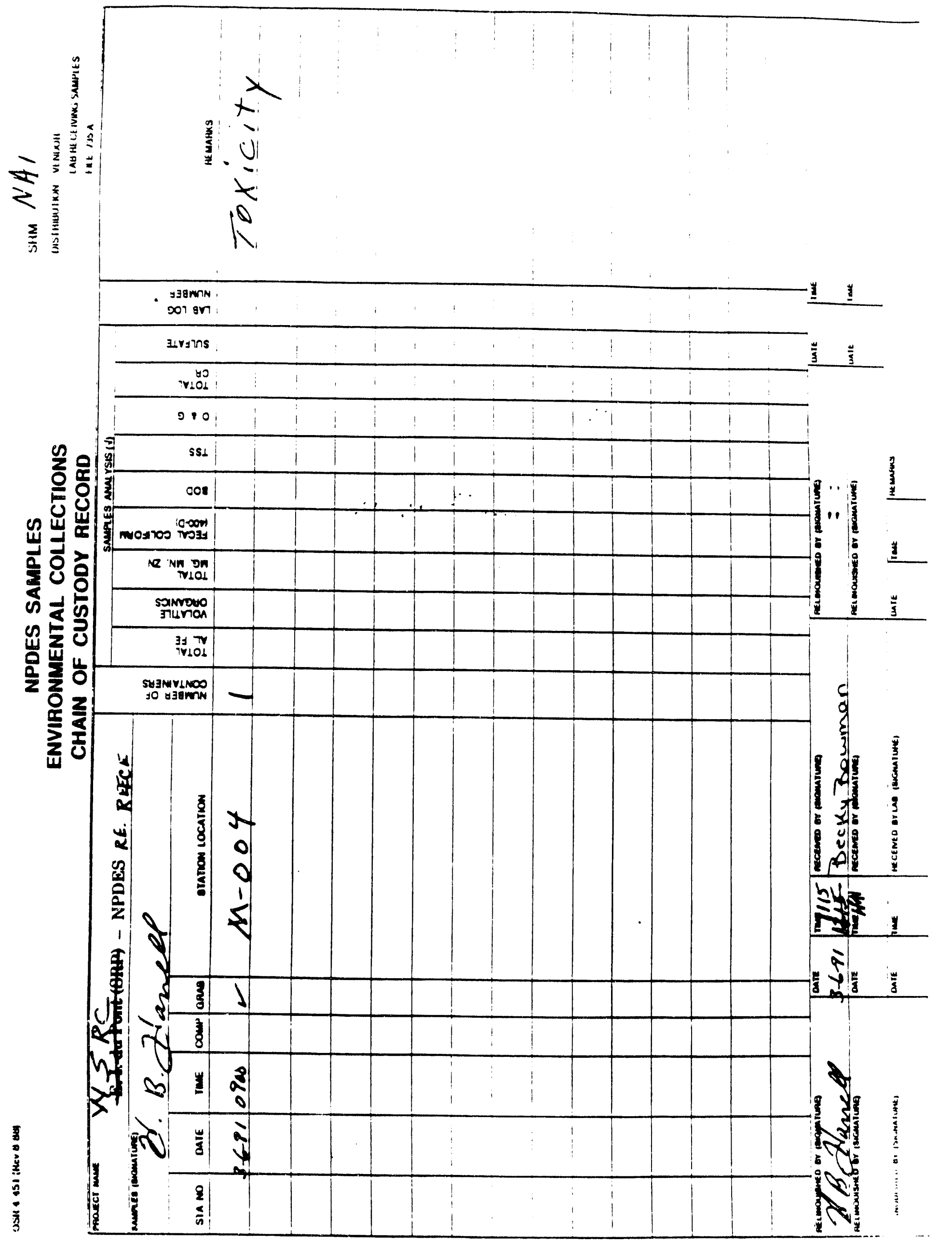


NPDES TOXICITY TEST REPORTING FORM - SCI

Facility:

Sampling Point:

NPDES \#:SC

Laboratory:

NORMANDEAU - SE

Receiving Stream:

Test organism: Ceriodaphria dubia remp.Range:

Test Type: [ ] hr acute; [ ] d chronic; static Started (date/time): h Ended (date/ti

Dilution water source zORO MIL Q: Per RIER

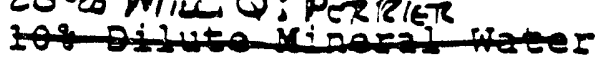

Sample Type: [ ] grab

Alk (mg/L) Hard (mg/L)

Sample $\neq$ Date/Time Collected
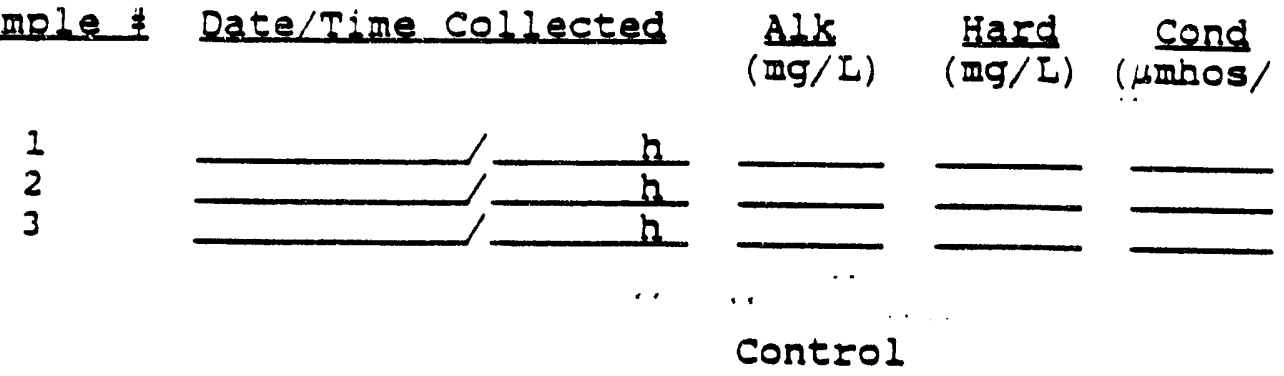

\begin{tabular}{|c|c|c|c|c|c|c|c}
\hline Day & & 0 & 1 & 2 & 3 & 4 & \\
\hline \multirow{3}{*}{ Temp } & Initial & & & & & & \\
\cline { 2 - 8 } Initial & & & & & & & \\
\cline { 2 - 8 } & Final & --- & & & & & \\
\cline { 2 - 8 } pH & Initial & & & & & & \\
\cline { 2 - 8 } & Final & -- & & & & & \\
\hline
\end{tabular}

\begin{tabular}{|c|c|c|c|c|c|c|c|}
\hline Day & & 0 & 1 & 2 & 3 & 4 & s \\
\hline \multirow[t]{2}{*}{ Temp } & Initial & & & & & & \\
\hline & Initial & & & & & & \\
\hline \multirow{2}{*}{ DO } & Final & $-\infty$ & & & & & \\
\hline & Initial & & & & & & \\
\hline $\mathrm{pH}$ & Final & --- & & & & & \\
\hline
\end{tabular}

Comments: «TRC measured in laboratory 

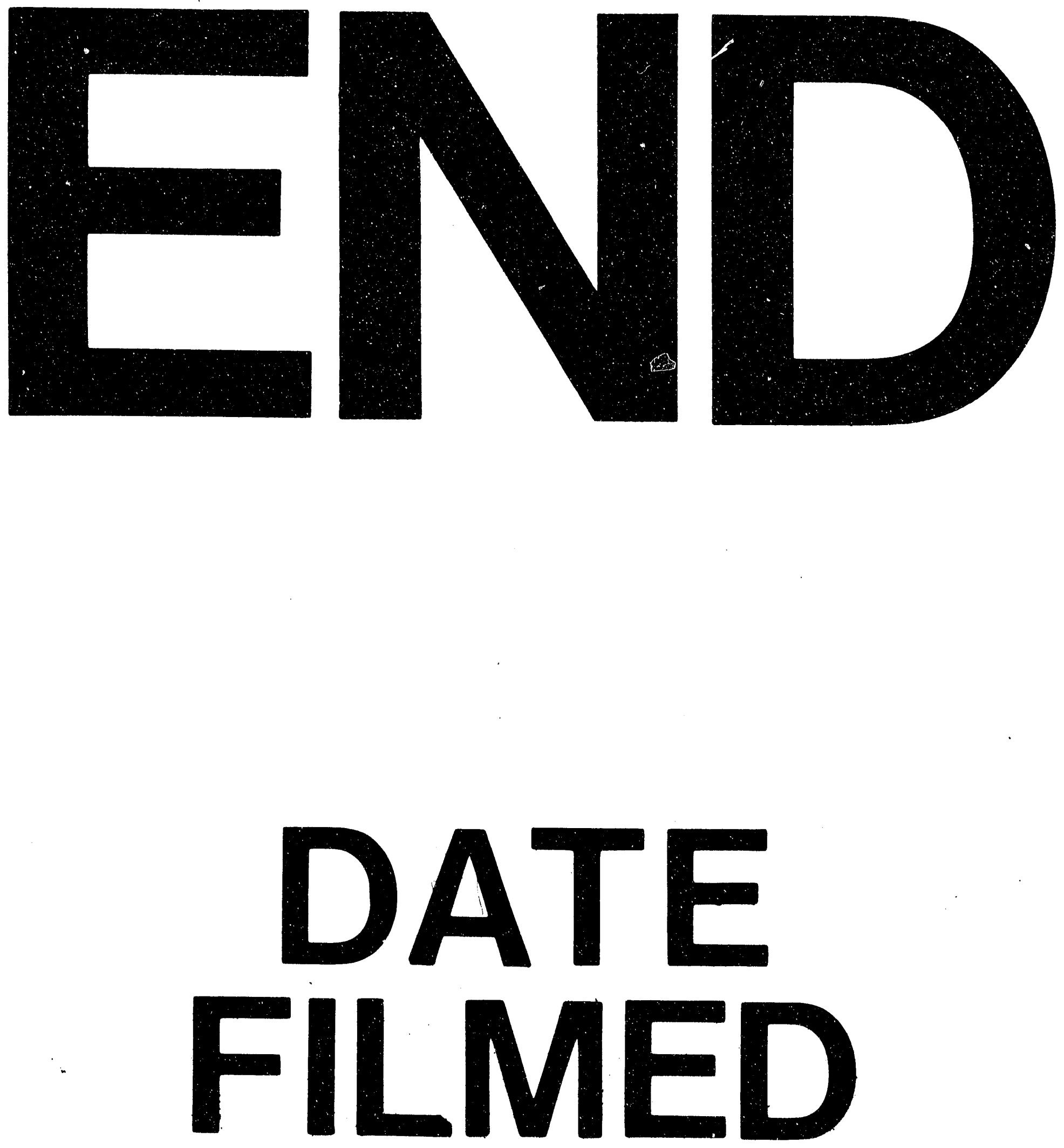

1

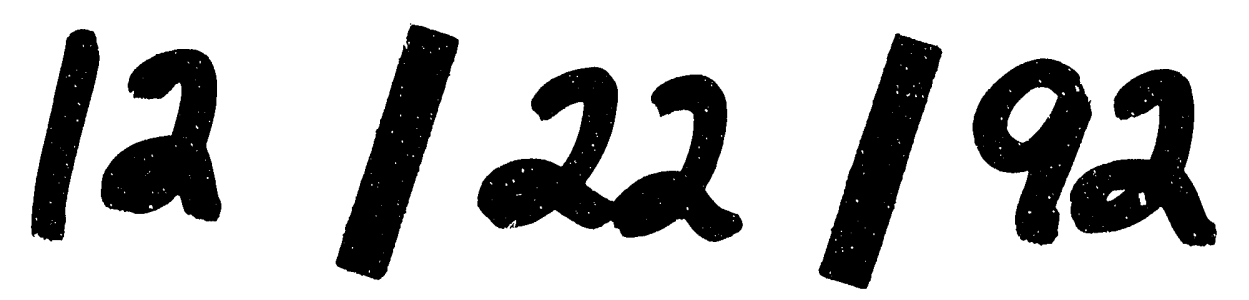


